\title{
Sheaf Theoretic Compactifications of the Space of Rational Quartic Plane Curves
}

\author{
Kiryong Chung
}

\begin{abstract}
Let $R_{4}$ be the space of rational plane curves of degree 4 . In this paper, we obtain a sheaf theoretic compactification of $R_{4}$ via the space of $\alpha$-semistable pairs on $\mathbb{P}^{2}$ and its birational relations through wall-crossings of semistable pairs. We obtain the Poincaré polynomial of the compactified space.
\end{abstract}

\section{Introduction}

\subsection{Compactifications and results}

Let $R_{d}$ be the space of irreducible rational plane curves of degree $d$. The space $R_{d}$ is an irreducible variety of dimension $3 d-1$ [16]. One of very famous compactification of $R_{d}$ is so called the moduli space of stable maps, which is a key role in the curve counting theory. For the detailed description, see [11]. This compactification is motivated by viewing the rational plane curve with its unique normalization map (up to isomorphism). In this paper, our aim is to obtain a sheaf-theoretic compactification of $R_{d}$ and its birational relations. In this case, the curve is regarded as the pair of the direct image sheaf of the normalizaition map and its canonical section. In detail,

(1) let $\mathrm{M}(d m+1)$ be the moduli space of stable sheaves $F$ with Hilbert polynomial $\chi(F(m))=d m+1$ and

(2) $\mathrm{M}^{\alpha}(d m+1)$ be the moduli space of $\alpha$-semistable pairs $(s, F)$ with Hilbert polynomial $\chi(F(m))=d m+1$.

For explicit definition and properties, see Section 2, Note that all of these moduli spaces are projective schemes $[14,17,21]$. For the irreducible plane curve $C$ of degree $d$, let $n: C^{v} \rightarrow C$ be the normalization map such that $C^{v} \cong \mathbb{P}^{1}$. Then the pair (resp. sheaf $\left.n_{*} \mathcal{O}_{C^{v}}\right)\left(s, n_{*} \mathcal{O}_{C^{v}}\right)$ with the unique section $s \in \mathrm{H}^{0}\left(n_{*} \mathcal{O}_{C^{v}}\right)$ is $\alpha$-stable (resp. stable) for any positive parameter $\alpha>0$ [2, Proposition 3.18]. That is, we have natural injective maps

$$
R_{d} \subset \mathrm{M}(d m+1) ; \quad[C] \mapsto n_{*} \mathcal{O}_{C^{v}} \quad \text { and } \quad R_{d} \subset \mathrm{M}^{\alpha}(d m+1) ; \quad[C] \mapsto\left(s, n_{*} \mathcal{O}_{C^{v}}\right) .
$$

Received August 6, 2020; Accepted January 11, 2021.

Communicated by Jungkai Alfred Chen.

2020 Mathematics Subject Classification. 14E15, 14E05, 14M15.

Key words and phrases. plane rational curves, compactification, birational geometry. 
Let us denote $\mathbf{M}_{d}$ (resp. $\left.\mathbf{M}_{d}^{\alpha}\right)$ by the closure of the rational curves space $R_{d}$ in the moduli space $\mathrm{M}(d m+1)\left(\right.$ resp. $\left.\mathrm{M}^{\alpha}(d m+1)\right)$.

Problem 1.1. Study the geometry $\mathbf{M}_{d}$ via its variations spaces $\mathbf{M}_{d}^{\alpha}$.

Remark 1.2. For the degree is $d \leq 3$, one can easily see that all of $\alpha$-stable sheaves spaces $\mathbf{M}_{d}^{\alpha}$ are isomorphic to $\mathbf{M}_{d}$. For $d=1$ and 2, the space $\mathbf{M}_{d}$ is isomorphic to the complete linear system $\left|\mathcal{O}_{\mathbb{P}^{2}}(d)\right|$. The space $\mathbf{M}_{3}$ is isomorphic to a $\mathbb{P}^{6}$-bundle over $\mathbb{P}^{2}$. Here the fiber $\mathbb{P}^{6}$ parameterizes the rational cubic curves passing through a singular point of $\mathbb{P}^{2}$.

For the higher degree cases since there does not have any systematic method to study the geometry of $\mathbf{M}_{d}$, we do the work for case $d=4$ firstly. We will prove that there exists a divisorial contraction among $\mathbf{M}_{d}^{\alpha}$. Let us state the main theorem of this paper. For sufficiently large parameter $\alpha \gg 1$, let denote by $\mathbf{M}_{d}^{\alpha \gg 1}:=\mathbf{M}_{d}^{+}$. It is known that the space $\mathbf{M}_{d}^{+}$is isomorphic to the relative Hilbert scheme of $(d-1)(d-2) / 2$-points over the complete linear system $\left|\mathcal{O}_{\mathbb{P}^{2}}(d)\right|$ (see [14, Section 4.4]). We call that a birational contraction is a smooth blow-down whenever the two birational varieties are locally smooth around the exceptional divisor and its image in the target space.

Theorem 1.3 (Proposition 3.6). Under the above notation, there exists a smooth blowdown

$$
p_{R}: \mathbf{M}_{4}^{+} \rightarrow \mathbf{M}_{4}
$$

where the exceptional divisor parameterizes the pair of the (degenerated) rational cubic curve and the collinear three points.

The key ingredient of the the proof of Theorem 1.3 is the analysis of the restrictions of pair wall-crossing of $\mathrm{M}^{\alpha}(4 m+1)$. Using this fact, one can compute the virtual Poincaré polynomial of the space $\mathbf{M}_{4}$ (see Corollary 4.4).

Corollary 1.4. The virtual Poincaré polynomial of $\mathbf{M}_{4}$ is given by

$$
1+2 t^{2}+5 t^{4}+8 t^{6}+11 t^{8}+12 t^{10}+13 t^{12}+13 t^{14}+11 t^{16}+7 t^{18}+3 t^{20}+t^{22} .
$$

Question 1.5. Find out a smooth resolution of the space $\mathbf{M}_{4}$ and study its geometry in the view point of curve counting theory.

In the introduction part of the paper [8], Diaz and Harris have already mentioned the necessity of a good compactification of the space $R_{d}$ for the intersection theory.

\subsection{Stream of the paper}

In Section 2, we explicitly propose relevant spaces and review its properties. In Section 3 , we prove our main theorem by analyzing the wall-crossing of $\alpha$-stable pairs on $\mathbb{P}^{2}$. In the last section, as presenting the special fibres by using Macaulay2 [12], we calculate the Poincaré polynomial of $\mathbf{M}_{4}$. 


\section{Relevant moduli spaces}

In this section, we collect known facts for the proof of Theorem 1.3

\subsection{Kronecker quiver and Hilbert scheme of cubes}

Let $\mathbf{N}:=\mathbf{N}(3 ; 2,3)$ be the moduli space of the space of sheaf homomorphisms (so called, the Kronecker quivers)

$$
\mathcal{O}_{\mathbb{P}^{2}}(-2)^{\oplus 2} \rightarrow \mathcal{O}_{\mathbb{P}^{2}}(-1)^{\oplus 3}
$$

up to the action of the automorphism group $\mathrm{GL}_{2} \times \mathrm{GL}_{3} / \mathbb{C}^{*}$. It is known that $\mathbf{N}$ is a smooth projective variety of dimension $6[9]$. On the other hand, let $Z$ be a finite subscheme of $\mathbb{P}^{2}$ of length 3 . Since a resolution of a general ideal sheaf $I_{Z}(1)$ twisted by $\mathcal{O}_{\mathbb{P}^{2}}(1)$ is of the form (2.1), the moduli space $\mathbf{N}$ is birational to the Hilbert scheme $\mathbf{H}[3]$ of three points in $\mathbb{P}^{2}$. The classification of locus in $\mathbf{H}[3]$ are very well-known. We list some loci which we will use later. Let $x, y, z$ be the homogeneous coordinates of $\mathbb{P}^{2}$.

(a) Let $D_{1}$ be the locus of collinear three points on $\mathbb{P}^{2}$ which is isomorphic to a $\mathbb{P}^{3}$-bundle over $\mathbb{P}^{2}$.

(b) Let $D_{2}$ be the locus of curvilinear three points which is isomorphic to $\mathbb{C}^{*} \times \mathbb{C}$-bundle over $\mathbb{P}^{2}$. The general element is of the form $\left\langle x^{2}, x y, y^{2}-z x\right\rangle$.

(c) Let $D_{3}:=\bar{D}_{2} \backslash D_{2}$ be the locus of the non-curvilinear three points which is isomorphic to $\mathbb{P}^{2}$. The general element is of the form $\left\langle x^{2}, x y, y^{2}\right\rangle$.

An explicit birational relation between $\mathbf{H}[3]$ and $\mathbf{N}$ has been studied in [18].

Proposition 2.1. [18, Section 6] Under the above notation, there exists a smooth blowdown morphism

$$
t: \mathbf{H}[3] \rightarrow \mathbf{N}
$$

which contracts the fiber $\mathbb{P}^{3}$ of the exceptional divisor $D_{1}$.

\subsection{The moduli of stable maps space}

Let $C$ be a projective connected reduced curve. A map $f: C \rightarrow \mathbb{P}^{r}$ is called by stable if $C$ has at worst nodal singularities and $|\operatorname{Aut}(f)|<\infty$. Let $\mathbf{M}\left(\mathbb{P}^{r}, d\right)$ be the moduli space of isomorphism classes of stable maps $f: C \rightarrow X$ with genus $g(C)=0$ and $\operatorname{deg}(f)=d$. The space $\mathbf{M}\left(\mathbb{P}^{r}, d\right)$ has been widely studied in [11]. In special, the space is compact and naturally contains the space $R_{d}$ as a dense open subset because the normalization map of an irreducible rational curve has the trivial automorphism. 


\subsection{Summary of the result in 4}

Let $X$ be a projective variety with a fixed ample line bundle $\mathcal{O}_{X}(1)$. Let

$$
\chi(F(m))=\sum_{k=0}^{\operatorname{dim} X}(-1)^{k} \operatorname{dim} \mathrm{H}^{k}(F(m))
$$

be the Hilbert polynomial of the coherent sheaf $F \in \operatorname{Coh}(X)$.

Definition 2.2. A pair $(s, F), s \in \mathrm{H}^{0}(F)$ is called $\alpha$-semistable if $F$ is pure and for any subsheaf $F^{\prime} \subset F$, the inequality

$$
\frac{\chi\left(F^{\prime}(m)\right)+\delta \cdot \alpha}{r\left(F^{\prime}\right)} \leq \frac{\chi(F(m))+\alpha}{r(F)}
$$

holds for $m \gg 0$. Here $\delta=1$ if the section $s$ factors through $F^{\prime}$ and $\delta=0$ otherwise. When the strict inequality holds, $(s, F)$ is called an $\alpha$-stable pair.

It is well-known that there exists a projective scheme $\mathrm{M}^{\alpha}(P(m))$ parameterizing $S$ equivalence classes of $\alpha$-semistable pairs with Hilbert polynomial $P(m)$ (see [17, Theorem 4.12] and [14, Theorem 2.6]). By decreasing the stability parameter $\alpha$, one can obtain a sequence of flips among the moduli spaces of $\alpha$-stable pairs.

As we let $\alpha=0$ in $(2.2)$, one can obtain the usual concept of semistable sheaves and thus the moduli space $\mathrm{M}(P(m))$ which parameterizes $S$-equivalence classes of semistable sheaves on $X$ with Hilbert polynomial $P(m)$ (For construction and its properties, see [21]). In this paper, we deal with the case $X=\mathbb{P}^{2}$ and $P(m)=4 m+1$. In [4,7], the authors studied the geometric properties of $\mathrm{M}^{\alpha}(4 m+1)$ and $\mathrm{M}(4 m+1)$ using the wall-crossing of pairs. It turns out that there exists a unique wall at $\alpha=3$ [4, Lemma 3.1]. Let us summarize the result for later use.

(i) (see [14, Section 4.4] and [20]) Let $\mathrm{M}^{+}(4 m+1):=\mathrm{M}^{\alpha}(4 m+1)$ for $\alpha>3$. The moduli space $\mathrm{M}^{+}(4 m+1)$ of $\alpha$-stable pairs is isomorphic to the relative Hilbert scheme $\mathcal{H}[3]$ of three points on the complete linear system $\left|\mathcal{O}_{\mathbb{P}^{2}}(4)\right|$. For $(s, F)$ be a stable pair, the corresponding point in the relative Hilbert scheme $\mathcal{H}[3]$ is given by

$$
\mathrm{M}^{+}(4 m+1) \rightarrow \mathcal{H}[3], \quad(s, F) \mapsto(Z, C), \quad Z \subset C=\operatorname{Supp}(F), l(Z)=3 .
$$

(ii) (see [4, Proposition 4.4]) Let $\mathrm{M}^{-}(4 m+1):=\mathrm{M}^{\alpha}(4 m+1)$ for $\alpha<3$. The forgetful map $r: \mathrm{M}^{-}(4 m+1) \rightarrow \mathrm{M}(4 m+1),(s, F) \mapsto F$ is a smooth blow-up along the Brill-Noether locus $\left\{[F] \in \mathrm{M}_{4} \mid \operatorname{dim} \mathrm{H}^{0}(F)=2\right\}$.

(iii) (see [4, Section 3.1]) The single flip over $\mathrm{M}^{3}(4 m+1)$ is a composition of a smooth blow-up and a smooth blow-down. Let us denote $Y^{+}$by the blow-up center in $\mathrm{M}^{+}$. 
Then $Y^{+}$is isomorphic to a $\mathbb{P}^{3}$-bundle over $\mathbb{P}^{2} \times \mathbb{P}^{9}$ where the base parameterizes the pair of line and cubic curves and the fiber parameterizes three points on that line.

Remark 2.3. The image of the blow-up center $Y^{-}$in $\mathrm{M}^{-}$by the forgetful map $r$ in (ii) parameterizes the stable sheaf $F$ which fits into a non-split extension:

$$
0 \rightarrow \mathcal{O}_{C} \rightarrow F \rightarrow \mathcal{O}_{L} \rightarrow 0
$$

for a line $L$ and a cubic curve $C$. By a simple computation, we see that the image $r\left(Y^{-}\right)$ is isomorphic to a $\mathbb{P}\left(\operatorname{Ext}_{\mathbb{P}^{2}}^{1}\left(\mathcal{O}_{L}, \mathcal{O}_{C}\right)\right) \cong \mathbb{P}^{2}$-bundle over $\mathbb{P}^{2} \times \mathbb{P}^{9}$. Also,

$$
h^{0}(F)= \begin{cases}1 & \text { if }[F] \in \mathbb{P}^{2} \backslash \mathbb{P}^{1}, \\ 2 & \text { if }[F] \in \mathbb{P}^{1}\end{cases}
$$

and thus $Y^{-} \cong r\left(Y^{-}\right)$by (ii).

In summary, there exists a commutative diagram

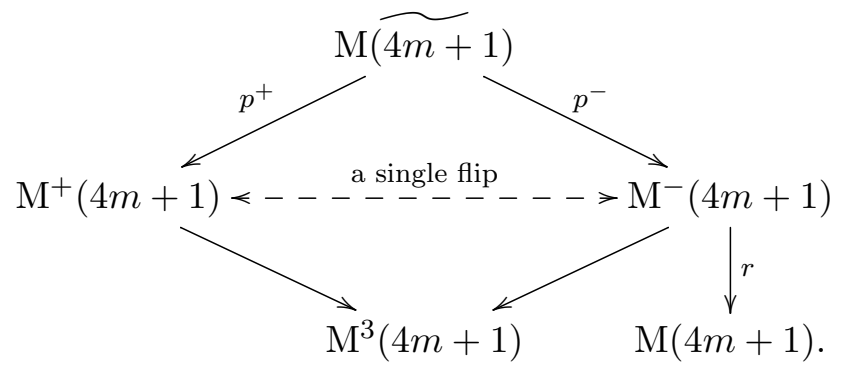

Let $\mathbf{M}_{4}^{\alpha}$ be the closure of the space $R_{4}$ of $\mathrm{M}_{4}^{\alpha}(4 m+1)$ for each $\alpha \in \mathbb{Q}^{+}$. Then the diagram (2.3) has been restricted to

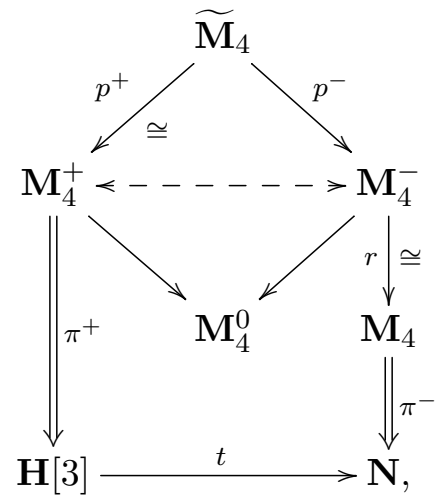

where $\pi^{+}: \mathbf{M}_{4}^{+} \rightarrow \mathbf{H}[3]$ is the restriction map of the structure morphism of the relative Hilbert scheme $\mathcal{H}[3] \rightarrow \mathbf{H}[3]$. The remaining one of this paper is to prove that 
- the restrictions of maps $p^{+}$and $r$ are an isomorphism,

- the composition of the restriction maps

$$
p_{R}:=r \circ p^{-} \circ\left(p^{+}\right)^{-1}: \mathbf{M}_{4}^{+} \rightarrow \mathbf{M}_{4}
$$

is a smooth blow-up map and

- the exists a generically bundle morphism $\pi^{-}: \mathbf{M}_{4} \rightarrow \mathbf{N}$ such that $\pi^{-} \circ p_{R}=t \circ \pi^{+}$. Remark 2.4. For the degree case $d \geq 5$, it is natural work to to find an explicit birational relation among the spaces $\mathbf{M}_{d}^{\alpha}$. It might be helpful to use a geometric description of the wall-crossings of $\mathrm{M}^{\alpha}(d m+1)$ for $d=5,6[3,4$.

\section{Proof of Theorem 1.3}

In this section, we analyze the restricted diagram in $(2.3)$ during the wall-crossing. One of main method is to use the elementary modification of sheaves coming from the family of stable maps.

\subsection{Some lemmas via stable maps}

Lemma 3.1. [2, Proposition 3.18] For the irreducible plane curve $C$, let $f: C^{v} \rightarrow C$ be the normalization map and $s \in \operatorname{Hom}\left(\mathcal{O}_{C}, f_{*} \mathcal{O}_{C^{v}}\right) \cong \mathrm{H}^{0}\left(\mathcal{O}_{C^{v}}\right)=\mathbb{C}$ be the canonical section. Then the pair $\left(s, f_{*} \mathcal{O}_{C^{v}}\right)$ is $\alpha$-stable for all $\alpha \in \mathbb{Q}^{+}$. In special, $f_{*} \mathcal{O}_{C^{v}}$ is stable.

The correspondence between $R_{4}$ and the relative Hilbert scheme $\mathcal{H}[3]$ is given by the following way. For the curve $C$, the direct image sheaf $f_{*} \mathcal{O}_{C^{v}}$ fits into the short exact sequence

$$
0 \rightarrow \mathcal{O}_{C} \rightarrow f_{*} \mathcal{O}_{C^{v}} \rightarrow \mathcal{Q} \rightarrow 0
$$

such that $\operatorname{dim} \mathcal{Q}=0$. By taking the dual $\mathcal{H o m} \bullet\left(-, \mathcal{O}_{C}\right)$ in the exact sequence,

$$
0 \rightarrow \mathcal{H o m}_{C}\left(f_{*} \mathcal{O}_{C^{v}}, \mathcal{O}_{C}\right) \rightarrow \mathcal{H o m}_{C}\left(\mathcal{O}_{C}, \mathcal{O}_{C}\right)=\mathcal{O}_{C} \rightarrow \mathcal{E} x t_{C}^{1}\left(\mathcal{Q}, \mathcal{O}_{C}\right) \rightarrow 0
$$

we have a pair $(Z, C), Z \subset C$ such that the subscheme $Z$ is defined by the (conductor) ideal sheaf $I_{Z, C}=\mathcal{H o m} C\left(f_{*} \mathcal{O}_{C}, \mathcal{O}_{C}\right)$ (cf. [20, Proposition B.5]).

Example 3.2. Let $f: \mathbb{P}^{1} \rightarrow \mathbb{P}^{2} ;[s: t] \mapsto\left[g_{3}(s, t) s: g_{3}(s, t) t: g_{4}(s, t)\right]$ be a map such that $g_{i}(s, t)$ are homogenous polynomial with respect to $s, t$ of degree $i$. Then it is straightforward to check that the conductor ideal sheaf is defined by

$$
I_{Z}=\left\langle x^{2}, x y, y^{2}\right\rangle
$$

where $x, y, z$ are homogenous coordinates of $\mathbb{P}^{2}$. 
Recall that $\mathbf{M}_{4}\left(\right.$ resp. $\left.\mathbf{M}_{4}^{\alpha}\right)$ is the closure of $R_{4}$ in $\mathrm{M}(4 m+1)\left(\right.$ resp. $\left.\mathbf{M}^{\alpha}(4 m+1)\right)$.

Proposition 3.3. For each closed point $[F] \in \mathbf{M}_{4}$,

$$
\operatorname{dim} \mathrm{H}^{0}(F)=1
$$

Hence the forgetful map $r$ is an isomorphism $\mathbf{M}_{4}^{-} \stackrel{r}{\cong} \mathbf{M}_{4}$.

Proof. Let $\mathcal{K}_{4}$ be the moduli space of stable maps of degree 4 into $\mathbb{P}^{2}$. Let

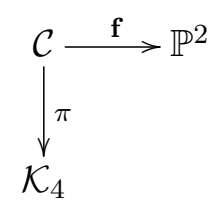

be a flat family of stable maps parameterized by $\mathcal{K}_{4}$. Let $\Phi:=(\pi, \mathbf{f}): \mathcal{C} \rightarrow \mathcal{K}_{4} \times \mathbb{P}^{2}$ be the push-out map. Then the direct image sheaf $\mathcal{F}:=\Phi_{*} \mathcal{O}_{\mathcal{C}}$ is the flat family of pure sheaves parameterized by $\mathcal{K}_{4}$. The general sheaves parameterized by $\mathcal{K}_{4}$ is stable by Lemma 3.1 . Also, $\mathrm{H}^{0}\left(\left.\mathcal{F}\right|_{[f] \times \mathbb{P}^{2}}\right) \cong \mathrm{H}^{0}\left(f_{*} \mathcal{O}_{C}\right)=\mathbb{C}$. The claim is proved by the below Lemma 3.4 .

Lemma 3.4. Let $S$ be a small disk in $\mathbb{C}$ containing 0 . Let $\mathcal{F}$ be a flat family of sheaves on

$$
\mathbb{P}^{r} \times S \rightarrow S
$$

with Hilbert polynomial $\chi(\mathcal{F}(m))=d m+1$. Let $\mathcal{F}$ be stable sheaves over $S$ except the origin $0 \in S$. Furthermore, $h^{0}\left(\left.\mathcal{F}\right|_{s}\right)=1$ for all $s \in S$. Then the modified stable sheaf at the origin has a unique non-zero global section.

Proof. Following the proof of [15, Theorem 2.B.1], it is enough to show that, after the single elementary modification of sheaf $\mathcal{F}$ along $0 \in S$, the resulting center fiber has a one-dimensional global section space. Let

$$
G \subset F:=\left.\mathcal{F}\right|_{0}
$$

be the non-zero maximal destabilizing subsheaf of $F$. Then we have a short exact sequence

$$
0 \rightarrow G \rightarrow F \rightarrow H:=F / G \rightarrow 0
$$

Let

$$
\chi(H(m))=d^{\prime} m+\chi^{\prime} \quad \text { and } \quad \chi(G(m))=\left(d-d^{\prime}\right) m+1-\chi^{\prime} .
$$

Then by the maximality of $G$, we have

$$
\frac{1-\chi^{\prime}}{d-d^{\prime}} \geq \frac{1}{d} \quad \Longleftrightarrow \quad \frac{d^{\prime}}{d} \geq \chi^{\prime}
$$


Since $h^{1}(F)=0$ and $G$ is supported on a curve in $\mathbb{P}^{r}, h^{1}(H)=0$. That is, $h^{0}(H)=\chi^{\prime} \geq 0$. Also, by above inequality, $\chi^{\prime} \leq 1$. If $\chi^{\prime}=1$, then it is contradict to the assumption of $G$. Thus $h^{0}(H)=0$. This implies that $h^{1}(G)=0$ because $h^{1}(F)=0$. Now, for the modified sheaf $\mathcal{F}^{\prime}$ on $\mathbb{P}^{r} \times S$, the central fiber fits into the short exact sequence

$$
\left.0 \rightarrow H \rightarrow \mathcal{F}^{\prime}\right|_{\mathbb{P}^{r} \times\{0\}} \rightarrow G \rightarrow 0
$$

Therefore $h^{1}\left(\left.\mathcal{F}^{\prime}\right|_{\mathbb{P}^{r} \times\{0\}}\right)=0$. That is, $h^{0}\left(\left.\mathcal{F}^{\prime}\right|_{\mathbb{P}^{r} \times\{0\}}\right)=1$.

\subsection{Proof of Theorem 1.3}

One of simple observation is that the canonical form of rational quartic plane curve with the prescribed singular points $([1: 0: 0],[0: 1: 0],[0: 0: 1])$ can be written as

$$
F(x, y, z)=a_{0} x^{2} y^{2}+a_{1} y^{2} z^{2}+a_{2} z^{2} x^{2}+a_{3} x^{2} y z+a_{4} x y^{2} z+a_{5} x y z^{2}
$$

where $x, y, z$ are the homogenous coordinate of $\mathbb{P}^{2}$ and $a_{0}, a_{1}, \ldots, a_{5} \in \mathbb{C}$. Hence the projection map $\pi^{+}: \mathbf{M}_{4}^{+} \rightarrow \mathbf{H}[3]$ in the diagram (2.4) is generically $\mathbb{P}^{5}$-fiberation over $\mathbf{H}[3]$.

Lemma 3.5. The map

$$
\pi^{+}: \mathbf{M}_{4}^{+} \rightarrow \mathbf{H}[3]
$$

has a $\mathbb{P}^{5}$-fibration over $\mathbf{H}[3] \backslash D_{1} \sqcup D_{3}$.

Proof. Let $\mathcal{Z}$ be the universal subscheme of $\mathbf{H}[3]$ and $q: \mathbf{H}[3] \times \mathbb{P}^{2} \rightarrow \mathbf{H}[3]$ be the projection onto the first component. Then, the push-forward sheaf $\mathcal{E}:=q_{*}\left(I_{\mathcal{Z}}^{2} \otimes \mathcal{O}_{\mathbb{P}^{2}}(4)\right)$ is locally free of rank 6 away from the union $D_{1} \sqcup D_{3}$. Indeed, it is straightforward to check that

$$
h^{0}\left(I_{Z}^{2}(4)\right)= \begin{cases}6 & \text { if }[Z] \in \mathbf{H}[3] \backslash D_{1} \sqcup D_{3}, \\ 7 & \text { if }[Z] \in D_{1}, \\ 7 & \text { if }[Z] \in D_{3}\end{cases}
$$

and thus the claim is proved. Now let $\mathbb{P}(\mathcal{E}):=\operatorname{Proj}(\operatorname{Sym} \bullet(\mathcal{E})) \rightarrow \mathbf{H}[3]$ be the projective scheme over $\mathbf{H}[3]$. Then $\mathbb{P}(\mathcal{E})$ is a subscheme of $\mathbf{M}_{4}^{+}$(cf. 14). Since $R_{4}$ is irreducible and the canonical form of rational quartic curve, $R_{4}$ is a subscheme of $\mathbb{P}(\mathcal{E})$. Thus $\mathbf{M}_{4}^{+}=$ $\overline{R_{4}} \subset \mathbb{P}(\mathcal{E})$. Therefore $\mathbf{M}_{4}^{+}$is also a $\mathbb{P}^{5}$-fiberation away from $D_{1} \sqcup D_{3}$.

Proposition 3.6. (1) The map

$$
\pi^{-}: \mathbf{M}_{4} \rightarrow \mathbf{N}
$$

is a $\mathbb{P}^{5}$-fiberation over $\mathbf{N} \backslash t\left(D_{3}\right)$. 
(2) Furthermore, the birational morphism $p_{R}: \mathbf{M}_{4}^{+} \rightarrow \mathbf{M}$ is a smooth blow-down of $\left(\pi^{+}\right)^{-1}\left(D_{1}\right)$ such that there exists a commutative diagram

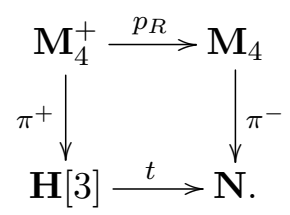

Proof. Recall that the image of the blow-up center $Y^{-}$by the forgetful map $r: \mathrm{M}^{-}(4 m+$ $1) \rightarrow \mathrm{M}(4 m+1)$ is isomorphic to a $\mathbb{P}^{2}$-bundle over $\mathbb{P}^{2} \times \mathbb{P}^{9}$ where the base space parameterizes the pair $(L, C)$ and the fiber is $\mathbb{P}\left(\operatorname{Ext}^{1}\left(\mathcal{O}_{L}, \mathcal{O}_{C}\right)\right)$ for the line $L$ and cubic curve $C$. Considering the image of the stable maps parameterized by $\mathcal{K}_{4}$, the support of the sheaf $F \in \mathbf{M} \cap r\left(Y^{-}\right)$must be $\operatorname{Supp}(F)=L \cup Q$ for some line $L$ and conic $Q$.

Step 1. Through the elementary modification of stable maps, we prove that

$$
\mathbf{M} \cap r\left(Y^{-}\right) \cong \mathbb{P}^{2} \times \mathbb{P}^{5}:=\Gamma
$$

where $\mathbb{P}^{2}$ (resp. $\mathbb{P}^{5}$ ) parameterizes lines (resp. coincs) in $\mathbb{P}^{2}$. Main stream of the proof is the same as that of [5, Proposition 5.10]. For the convenience of reader we address the detail. Let $\Delta$ be the locus of the stable maps $f: C \rightarrow \mathbb{P}^{2}$ of degree 4 such that the image is $f(C)=L \cup Q$ where $L$ (resp. $Q$ ) is a line (resp. conic). One can regard $f$ as the composition map

$$
f=g \circ h: C \stackrel{h}{\rightarrow} C^{\prime} \stackrel{g}{\rightarrow} \mathbb{P}^{2},
$$

where $C^{\prime}$ is a pair of lines. Here $h$ is a $2: 1$-covering from $L_{1}$ and a bijection from $L_{2}$. Also, $g$ is a partial normalization map such that the image is $g\left(C^{\prime}\right)=L \cup Q$. By applying the octahedron axiom for the complex $f^{*} \Omega_{\mathbb{P}^{2}} \rightarrow h^{*} \Omega_{C^{\prime}} \rightarrow \Omega_{C}$, we see that the normal space of $\Delta$ in $\mathbf{M}\left(\mathbb{P}^{2}, 4\right)$ at $[f]$ is identified with

$$
N_{\Delta / \mathbf{M}\left(\mathbb{P}^{2}, 4\right),[f]} \cong \operatorname{Ext}_{C^{\prime}}^{1}\left(\left[g^{*} \Omega_{\mathbb{P}^{2}} \rightarrow \Omega_{C^{\prime}}\right], O_{L}(-1)\right) .
$$

On the other hand, let $i: L_{2} \subset C^{\prime}$ be the inclusion map. By the octahedron axiom for the complex $(i \circ g)^{*} \Omega_{\mathbb{P}^{2}} \rightarrow i^{*} \Omega_{C^{\prime}} \rightarrow \Omega_{L_{2}}$ again, one can easily see that there exists a quasi-isomorphism between

$$
i^{*}\left[g^{*} \Omega_{\mathbb{P}^{2}} \rightarrow \Omega_{C^{\prime}}\right] \cong N_{L / \mathbb{P}^{2}}^{*}(-1)[1]
$$

and thus

$$
N_{\Delta / \mathbf{M}\left(\mathbb{P}^{2}, 4\right),[f]} \cong \operatorname{Hom}\left(N_{L / \mathbb{P}^{2}}^{*}(-1), \mathcal{O}_{L}(-1)\right) \cong \operatorname{Hom}\left(I_{L}(-1), \mathcal{O}_{L}(-1)\right) .
$$

By analyzing the Kodaira-Spencer map

$$
\phi: N_{\Delta / \mathbf{M}\left(\mathbb{P}^{2}, 4\right),[f]} \rightarrow \operatorname{Ext}^{1}\left(g_{*} \mathcal{O}_{C^{\prime}}, \mathcal{O}_{L}(-1)\right),
$$


one can prove that $\phi$ is injective by using the short exact sequence $0 \rightarrow \mathcal{O}_{Q}(-p) \rightarrow$ $g_{*} \mathcal{O}_{C^{\prime}} \rightarrow \mathcal{O}_{L} \rightarrow 0, p=L \cap Q \backslash f\left(L_{1} \cap L_{2}\right)$ and thus each modified sheaf is non-split (cf. 6, (4.10)]). Hence after the modification, we have a flat family of stable sheaves parameterized by $\mathbb{P}\left(N_{\Delta / \mathbf{M}\left(\mathbb{P}^{2}, 4\right)}\right)=\mathbb{P}^{1}$-bundle over $\Delta$. For $w \in \mathbb{P}^{1}$, let $F_{w}$ be the stable sheaf which fits into a exact sequence

$$
0 \rightarrow \mathcal{O}_{L}(-1) \rightarrow F_{w} \rightarrow g_{*} \mathcal{O}_{C^{\prime}} \rightarrow 0
$$

By Proposition 3.3, $h^{0}\left(F_{w}\right)=1$ for each point $[w] \in \mathbb{P}^{1}$. Hence $F_{w} \cong F_{w^{\prime}}$ for any point $[w],\left[w^{\prime}\right] \in \mathbb{P}^{1}$ by Bézout's Theorem (see Remark 2.3). By some diagram chasing, we know that $F_{w}$ fits into the short exact sequence

$$
0 \rightarrow \mathcal{O}_{L \cup Q} \rightarrow F_{w} \rightarrow \mathcal{O}_{L} \rightarrow 0
$$

and thus the claim is proved. Hence the composition map $t \circ \pi^{+} \circ p^{+}: \widetilde{\mathrm{M}(\widetilde{4 m+1})} \rightarrow \mathbf{N}$ factors through $\mathbf{M}_{4}^{-}=\mathbf{M}_{4}$ by the rigidity lemma. That is, we have a morphism

$$
\pi^{-}: \mathbf{M}_{4} \rightarrow \mathbf{N}
$$

Note that the fiber of $[L] \in t\left(D_{1}\right) \subset \mathbf{N}$ is constantly a $\mathbb{P}^{5}$ which parameterizes conics. By Lemma 3.5 , we know that the map $\pi^{-}$is a $\mathbb{P}^{5}$-fiberation except the locus $D_{3}$.

Step 2. By (1), the normal space of $\Gamma$ in $\mathbf{M}_{4}$ at $\left[F_{w}\right] \in \mathbf{M}_{4}$ is naturally isomorphic to

$$
N_{\Gamma / \mathbf{M}_{4}, F_{w}} \cong N_{t\left(D_{1}\right) / \mathbf{N},\left[\pi^{-}\left(F_{w}\right)\right]}
$$

This implies that the blow-up of $\mathbf{M}_{4}=\mathbf{M}_{4}^{+}$along $\Gamma$ is nothing but the strict transformation of the blow-up morphism $p^{-}: \mathrm{M}(4 m+1) \rightarrow \mathrm{M}^{-}(4 m+1)$ because the projectivation of the normal space $N_{t\left(D_{1}\right) / \mathbf{N},\left[\pi^{-}\left(F_{w}\right)\right]}$ determines three points on the line $L$. In special, the exceptional divisor $E$ in $\widetilde{\mathbf{M}}^{+}$is a $\mathbb{P}^{3}$-bundle over $\Gamma$. Then the image $p^{+}(E) \cong E \subset Y^{+}$is still divisor in $\mathbf{M}^{+}$and thus $\widetilde{\mathbf{M}}+\stackrel{p^{+}}{\cong} \mathbf{M}^{+}$. Note that $E$ is the inverse image $\left(\pi^{+}\right)^{-1}\left(D_{1}\right)$ by its construction. This finishes the proof of (2).

\section{Poincaré polynomial of $\mathbf{M}_{4}$}

In this section we compute the Poincaré polynomial of $\mathbf{M}_{4}$ by using Proposition 3.6 .

\subsection{Fibre over the non-curvilinear points}

In this section, we describe the fiber of the morphism $p$ over $Y_{1}$ by using the computer algebra system, Macaulay2 [12] and hence we compute the virtual Poincaré polynomial of $\mathrm{M}_{4}$. 
Proposition 4.1. Each fiber $\pi^{-}: \mathbf{M}_{4} \rightarrow \mathbf{N}$ over the closed point $[Z] \in D_{3}$ is isomorphic to a projective space $\mathbb{P}^{8}$.

Proof. We assume that $I_{Z}=\left\langle x^{2}, x y, y^{2}\right\rangle$. We prove the claim by presenting a local neighborhood of $\mathbf{M}_{4}^{+}$containing the fiber $p^{-1}([Z])$ with the help of the computer system. Let $a_{1}$, $a_{2}, b_{1}, b_{2}, c_{1}, c_{2}$ be a local affine chart of $\mathbf{H}[3]$ at $\left[I_{Z}\right]=\left[\left\langle x^{2}, x y, y^{2}\right\rangle\right]$ (see 13 , Section 3.1]). The ideal of subschemes parameterizing the points of $\mathbf{H}[3]$ around $[Z]$ is generated by three quadric polynomials:

(i) $q_{1}:=x^{2}-a_{1} x-a_{2} y-a_{2}\left(b_{1}-c_{2}\right)-b_{2}\left(b_{2}-a_{1}\right)$;

(ii) $q_{2}:=x y-b_{1} x-b_{2} y-a_{2} c_{1}+b_{1} b_{2}$;

(iii) $q_{3}:=y^{2}-c_{1} x-c_{2} y-c_{1}\left(b_{2}-a_{1}\right)-b_{1}\left(b_{1}-c_{2}\right)$.

Note that $\left.\left\langle q_{1}, q_{2}, q_{3}\right\rangle\right|_{\left\{a_{i}=b_{i}=c_{i}=0\right\}}=I_{Z}$ for $i=1,2$. Let

$$
\begin{aligned}
f(x, y, z)= & d_{0} x^{4}+d_{1} x^{3} y+d_{2} x^{2} y^{2}+d_{3} x y^{3}+\cdots+d_{8} y^{3} z+d_{9} x^{2} z^{2}+d_{10} y^{2} z^{2} \\
& +d_{11} x y z^{2}+d_{12} x z^{3}+d_{13} y z^{3}+d_{14} z^{4}
\end{aligned}
$$

be the homogeneous polynomial defining the plane quartic curve. Let $f_{x}$ be the partial derivatives of $f$ with respect to $x$ (similarly, $f_{y}$ and $f_{z}$ ). The rationality condition of the plane curve provides us the nine resultant conditions

$$
J=\left\langle\operatorname{Res}\left(f_{x}, q_{i}\right), \operatorname{Res}\left(f_{y}, q_{i}\right), \operatorname{Res}\left(f_{z}, q_{i}\right)\right\rangle \text { for } i=1,2,3 \text {. }
$$

Note that $f_{x}(x, y, 1) \equiv s(x, y) \bmod \left(q_{1}, q_{2}, q_{3}\right)$ for some quadric polynomial $s(x, y)$ (similarly, $f_{y}(x, y, 1)$ and $\left.f_{z}(x, y, 1)\right)$. Hence the resultant can be easily computed by using the computer algebra system, Macaulay2 [12] and obtain that the ideal $J$ is the defining equation of $\mathbf{M}^{+}$around $\left(\pi^{+}\right)^{-1}([Z])$. The special fiber $\left(\pi^{+}\right)^{-1}([Z])$ is nothing but

$$
J+\left\langle a_{1}, a_{2}, b_{1}, b_{2}, c_{1}, c_{2}\right\rangle=\left\langle d_{9}, d_{10}, d_{11}, d_{12}, d_{13}, d_{14}\right\rangle
$$

which defines the space $\mathbb{P}^{8}$. Thus we finish the proof of the claim.

Remark 4.2. The fiber $[Z]$ by $\pi^{-}$in the statement of Proposition 4.1 can be described by the stable map. As we have seen in Example 3.2 , the conductor ideal of the map

$$
f: \mathbb{P}^{1} \rightarrow \mathbb{P}^{2}, \quad f([s: t])=\left[g_{3}(s, t) s: g_{3}(s, t) t: g_{4}(s, t)\right]
$$

is given by $\left\langle x^{2}, x y, y^{2}\right\rangle$ for the general choice of $g_{3}(s, t)$ and $g_{4}(s, t)$. Also the supporting curve $C=f\left(\mathbb{P}^{1}\right)$ is defined by the ideal $I_{C}=\left\langle g_{3}(x, y) z-g_{4}(x, y)\right\rangle$. Hence the fiber $p^{-1}([Z])$ contains an open part of $\mathbb{P}\left(\mathrm{H}^{0}\left(\mathcal{O}_{\mathbb{P}^{1}}(3) \oplus \mathcal{O}_{\mathbb{P}^{1}}(4)\right)\right)$. 


\subsection{The virtual Poincaré polynomial of $\mathbf{M}_{4}$}

In this subsection, we calculate the virtual Poincaré polynomial of $\mathbf{M}$ by using Theorem 1.3. Let $X$ be a quasi-projective variety. For the Hodge-Deligne polynomial $\mathrm{E}_{c}(X)(u, v)$ for compactly supported cohomology of $X$, let

$$
\mathrm{P}(X):=\mathrm{E}_{c}(X)(-t,-t)
$$

be the virtual Poincaré polynomial of $X$. For the motivic properties of the virtual Poincaré polynomial, see [19, Theorem 2.2] and [1, Lemma 3.1].

Proposition 4.3. (1) $\mathrm{P}\left(\mathbb{P}^{n}\right)=\left(t^{2 n+2}-1\right) /\left(t^{2}-1\right)$.

(2) $\mathrm{P}(X)=\mathrm{P}(Z)+\mathrm{P}(X \backslash Z)$ for any closed subset $Z \subset X$.

(3) $\mathrm{P}(X)=\mathrm{P}(F) \cdot \mathrm{P}(B)$ for the étal locally trivial fiberation $X \rightarrow B$ with the constant fiber isomorphic to the Grassmannian variety $\operatorname{Gr}(k, n)$. Here $\operatorname{Gr}(k, n)$ parameterizes $k$-dimensional subspaces in the vector space $\mathbb{C}^{n}$.

Corollary 4.4. The virtual Poincaré polynomial of $\mathbf{M}_{4}$ is given by

$$
1+2 t^{2}+5 t^{4}+8 t^{6}+11 t^{8}+12 t^{10}+13 t^{12}+13 t^{14}+11 t^{16}+7 t^{18}+3 t^{20}+t^{22} .
$$

Proof. By $P(\mathbf{H}[3])=1+2 t^{2}+5 t^{4}+6 t^{6}+5 t^{8}+2 t^{10}+t^{12}[10$ and Proposition 2.1.

$$
P(\mathbf{N})=P(\mathbf{H}[3])+\left(1-P\left(\mathbb{P}^{3}\right)\right) \cdot P\left(\mathbb{P}^{2}\right)=1+t^{2}+3 t^{4}+3 t^{6}+3 t^{8}+t^{10}+t^{12} .
$$

Also, from Propositions 3.6, 4.1 and 4.3, we have

$$
P\left(\mathbf{M}_{4}\right)=P\left(\mathbb{P}^{5}\right) \cdot\left(P(\mathbf{N})-P\left(\mathbb{P}^{2}\right)\right)+P\left(\mathbb{P}^{8}\right) \cdot P\left(\mathbb{P}^{2}\right) .
$$

Cooking up these ones, we obtain the results.

Remark 4.5. The result of Corollary 4.4 can be also obtained by using Lemma 3.5 and the birational map $p_{R}$ in Proposition 3.6(2).

\section{Acknowledgments}

The author would like to thank Dawei Chen for suggesting this topic and Jinhyung Park for helpful discussions and comments. Kiryong Chung is partially supported by Korea NRF grant 2019R1F1A1042516. 


\section{References}

[1] B. Bakker and A. Jorza, Higher rank stable pairs on K3 surfaces, Commun. Number Theory Phys. 6 (2012), no. 4, 805-847.

[2] D. Chen and I. Coskun, Towards Mori's program for the moduli space of stable maps, Amer. J. Math. 133 (2011), no. 5, 1389-1419.

[3] J. Choi and K. Chung, The geometry of the moduli space of one-dimensional sheaves, Sci. China Math. 58 (2015), no. 3, 487-500.

[4] _ Moduli spaces of $\alpha$-stable pairs and wall-crossing on $\mathbb{P}^{2}$, J. Math. Soc. Japan 68 (2016), no. 2, 685-709.

[5] J. Choi, K. Chung and M. Maican, Moduli of sheaves supported on quartic space curves, Michigan Math. J. 65 (2016), no. 3, 637-671.

[6] K. Chung and Y.-H. Kiem, Hilbert scheme of rational cubic curves via stable maps, Amer. J. Math. 133 (2011), no. 3, 797-834.

[7] K. Chung and H.-B. Moon, Chow ring of the moduli space of stable sheaves supported on quartic curves, Q. J. Math. 68 (2017), no. 3, 851-887.

[8] S. Diaz and J. Harris, Geometry of the Severi variety, Trans. Amer. Math. Soc. 309 (1988), no. 1, 1-34.

[9] J.-M. Drezet, Fibrés exceptionnels et variétés de modules de faisceaux semi-stables $\operatorname{sur} \mathbf{P}_{2}(\mathbf{C})$, J. Reine Angew. Math. 380 (1987), 14-58.

[10] G. Elencwajg and P. Le Barz, Explicit computations in $\mathrm{Hilb}^{3} \mathbf{P}^{2}$, in: Algebraic Geometry (Sundance, UT, 1986), 76-100, Lecture Notes in Math. 1311, Springer, Berlin, 1988.

[11] W. Fulton and R. Pandharipande, Notes on stable maps and quantum cohomology, in: Algebraic Geometry (Santa Cruz 1995), 45-96, Proc. Sympos. Pure Math. 62 Part 2, Amer. Math. Soc., Providence, RI, 1997.

[12] D. R. Grayson and M. E. Stillman, Macaulay2: A software system for research in algebraic geometry, Available at https://faculty.math.illinois.edu/Macaulay2/.

[13] M. Haiman, Notes on Macdonald polynomials and the geometry of Hilbert schemes, in: Symmetric Functions 2001: Surveys of Developments and Perspectives, 1-64, MATO Sci. Ser. II Math. Phys. Chem. 74, Kluwer Acad. Publ., Dordrecht, 2002. 
[14] M. He, Espaces de modules de systèmes cohérents, Internat. J. Math. 9 (1998), no. 5, $545-598$.

[15] D. Huybrechts and M. Lehn, The Geometry of Moduli Spaces of Sheaves, Second edition, Cambridge Mathematical Library, Cambridge University Press, Cambridge, 2010.

[16] B. Kim and R. Pandharipande, The connectedness of the moduli space of maps to homogeneous spaces, in: Symplectic Geometry and Mirror Symmetry (Seoul, 2000), 187-201, World Sci. Publ., River Edge, NJ, 2001.

[17] J. Le Potier, Systèmes cohérents et structures de niveau, Astérisque 214 (1993), 143 pp.

[18] W.-P. Li, Z. Qin and Q. Zhang, Curves in the Hilbert schemes of points on surfaces, in: Vector Bundles and Representation Theory (Columbia, MO, 2002), 89-96, Contemp. Math. 322, Amer. Math. Soc., Providence, RI, 2003.

[19] V. Muñoz, Hodge polynomials of the moduli spaces of rank 3 pairs, Geom. Dedicata 136 (2008), 17-46.

[20] R. Pandharipande and R. P. Thomas, Stable pairs and BPS invariants, J. Amer. Math. Soc. 23 (2010), no. 1, 267-297.

[21] C. T. Simpson, Moduli of representations of the fundamental group of a smooth projective variety I, Inst. Hautes Études Sci. Publ. Math. 79 (1994), 47-129.

Kiryong Chung

Department of Mathematics Education, Kyungpook National University, 80 Daehakro,

Bukgu, Daegu 41566, Korea

E-mail address: krchung@knu.ac.kr 